\title{
OCORRÊNCIA DE OPHIOMORPHA NODOSA EM SEDIMENTOS PLEISTOCÊNICOS DA PLANÍCIE COSTEIRA DA PINHEIRA, SANTA CATARINA, BRASIL
}

\author{
DANYELLY CAETANO MARTINS \\ Departamento de Geociências, Universidade Federal de Santa Catarina, Campus Universitário, s/n, 88040-900, \\ Florianópolis, Santa Catarina, Brasil.danycaetanomartins@gmail.com
}

RODRIGO R. CANCELLI

Centro de Estudos de Geologia Costeira e Oceânica, Instituto de Geociências, Universidade Federal do Rio Grande do Sul, Campus do Vale, Av. Bento Gonçalves, 9500, 91501-970, Porto Alegre, Rio Grande do Sul, Brasil. rodrigocancelli@hotmail.com

RENATO PEREIRA LOPES

Universidade Federal do Pampa, Campus Caçapava do Sul, Av. Pedro Anunciação, 111, 96570-000, Vila Batista, Caçapava do Sul, Rio Grande do Sul, Brasil.paleonto_furg@yahoo.com.br

\section{PATRÍCIA HADLER, ERICKS H. TESTA}

Laboratório de Paleontologia, Departamento de Geociências, Universidade Federal de Santa Catarina, Campus Universitário, s/n, 88040-900, Florianópolis, Santa Catarina, Brasil.patricia.hadler@ufsc.br,ericks.testa@gmail.com

\section{EDUARDO GUIMARÃES BARBOZA}

Centro de Estudos de Geologia Costeira e Oceânica, Instituto de Geociências, Universidade Federal do Rio Grande do Sul, Campus do Vale, Av. Bento Gonçalves, 9500, 91501-970, Porto Alegre, Rio Grande do Sul, Brasil. eduardo.barboza@ufrgs.br

\begin{abstract}
Occurrence of Ophiomorpha nodosa in Pleistocene sediments of Coastal Plain of Pinheira, Santa Catarina, Brazil. The occurrence of Pleistocene (MIS) 5 marine ichnofossils in a locality of the Coastal Plain of Pinheira, State of Santa Catarina is described, and their value as indicators of the geological/stratigraphical evolution in this area is discussed. This sedimentary sequence indicates the transition among the subtidal zone, where the ichnofossils occur, to the supratidal zone, and finally to dunes. The ichnofossils can be seen in vertical view, between 0 and $4.5 \mathrm{~m}$ above the present sea level. The galleries are cylindrical structures with circular or oval transversal section, without ramifications. Their interior walls are smooth, and the exterior walls are composed of pellets made of clay and sand homogeneously dispersed, without a specific arrangement. This morphology is related to ichnospecies Ophiomorpha nodosa. The average density was 32.9 galleries/ $\mathrm{m}^{2}$, ranging from 22 to 51 galleries $/ \mathrm{m}^{2}$. The highest density found could be related to time-averaging, or to a deeper subtidal environment. The vertical distribuition of Ophiomorpha and the absence of faciological variations in the deposits might indicate an aggradational stage between the trangressive and regressive phases.
\end{abstract}

Keywords: Ophiomorpha, ichnofossils, Pleistocene, paleo-sea level.

RESUMO - A ocorrência de icnofósseis marinhos do Pleistoceno (MIS) 5 em uma localidade da Planície Costeira da Pinheira, Estado de Santa Catarina é descrita, e seu valor como indicador da evolução geológica/estratigráfica nesta área é discutido. Esta sequência sedimentar indica a transição entre a zona de inframaré, onde os icnofósseis ocorrem, para zona de supramaré e, finalmente para dunas. Os icnofósseis podem ser observados em posição vertical, entre 0 e 4,5 m acima do nível do mar atual. As galerias são estruturas cilíndricas com seção transversal circular ou oval, sem ramificações. As paredes internas são lisas, e as externas são compostas por pellets de argila e areia homogeneamente dispersos, sem um arranjo específico. Esta morfologia está relacionada com a icnoespécie Ophiomorpha nodosa. A densidade média foi de 32,9 galerias $/ \mathrm{m}^{2}$, variando de 22 a 51 galerias $/ \mathrm{m}^{2}$. A maior densidade encontrada pode estar relacionada com time-averaging, ou com um ambiente de inframaré mais profundo. A distribuição vertical de Ophiomorpha e a ausência de variações faciológicas nos depósitos podem indicar um estágio agradacional entre as fases transgressiva e regressiva.

Palavras-chave: Ophiomorpha, icnofósseis, Pleistoceno, paleonível marinho. 


\section{INTRODUÇÃO}

O icnogênero Ophiomorpha engloba estruturas de habitação produzidas em substratos arenosos na forma de galerias cilíndricas verticais, que se estendem entre 2 e $4 \mathrm{~m}$ abaixo da superfície, onde se ramificam em galerias horizontais e inclinadas. As galerias são lisas internamente e têm a parede externa formada por pellets subesféricos a elípticos feitos de areia cimentada com argilominerais, óxido e sulfeto de ferro, fosfato de cálcio amorfo ou cristobalita secretada pelo organismo construtor (Weimer \& Hoyt, 1964; Kennedy \& MacDougall, 1969; Dike, 1972; Häntzschel, 1975; Frey et al., 1978). A origem destes icnitos tem sido atribuída a decápodes marinhos da família Callianassidae como, por exemplo, o gênero Callichirus (Weimer \& Hoyt, 1964; Häntzschel, 1975; Fernandes et al., 2002).

Embora Ophiomorpha seja considerado um importante indicador paleoambiental, apontado como referência para a determinação de paleonível marinho e da posição de antigas linhas de costa, por ocupar ambientes costeiros rasos (e.g. Howard, 1972; Tomazelli et al., 1982; Gibert et al., 2006; Tomazelli \& Dillenburg, 2007; Gandini \& Netto, 2012; Netto et al., 2012; Angulo \& Souza, 2014; Lopes et al., 2014), este icnogênero engloba galerias produzidas por invertebrados que habitam desde lagunas e baías até praias de alta energia e áreas offshore (Bromley \& Frey, 1974; Pryor, 1975; Frey et al., 1978; Stewart, 1978).
A distribuição estratigráfica de Ophiomorpha no Brasil vai do Permiano ao Quaternário. A icnoespécie Ophiomorpha nodosa está registrada na transição Cretáceo-Paleógeno da Paraíba (Barboza et al., 2006), no Mioceno do Acre, Alagoas e Maranhão (Gingras et al., 2002; Netto \& Rossetti, 2003; Rossetti \& Góes, 2009), no Pleistoceno do Rio Grande do Norte, Bahia, São Paulo e Rio Grande do Sul (Suguio \& Martin, 1976; Barreto et al., 2002; Gibert et al., 2006; Tomazelli \& Dillenburg, 2007; Dominguez \& Bittencourt, 2009; Suguio et al., 2011; Lopes et al., 2014; Rosa et al., 2017), e em sedimentos holocênicos do Rio Grande do Norte (Vieira et al., 2007). Outra icnoespécie, O. puerilis, tem sido registrada em sedimentos marinhos pleistocênicos da "Formação Chuí" (Barreira III de Villwock et al., 1986) no sul do Brasil (Gibert et al., 2006; Netto et al., 2017).

No Estado de Santa Catarina, há apenas dois registros prévios do icnogênero Ophiomorpha, um nas proximidades da cidade de Paulo Lopes, adjacente ao afloramento aqui estudado (Caruso Jr. et al., 1999), e outro na Ilha de São Francisco do Sul, região norte do estado (Horn Filho \& Simó, 2008; Vieira et al., 2011). Estas ocorrências foram utilizadas para inferências paleoambientais, sem maiores detalhes sobre os icnofósseis.

Aqui é apresentada uma descrição dos espécimes de Ophiomorpha nodosa encontrados em sedimentos pleistocênicos próximo à Praia da Pinheira, englobando dados de suas medidas, densidade e distribuição populacional, bem
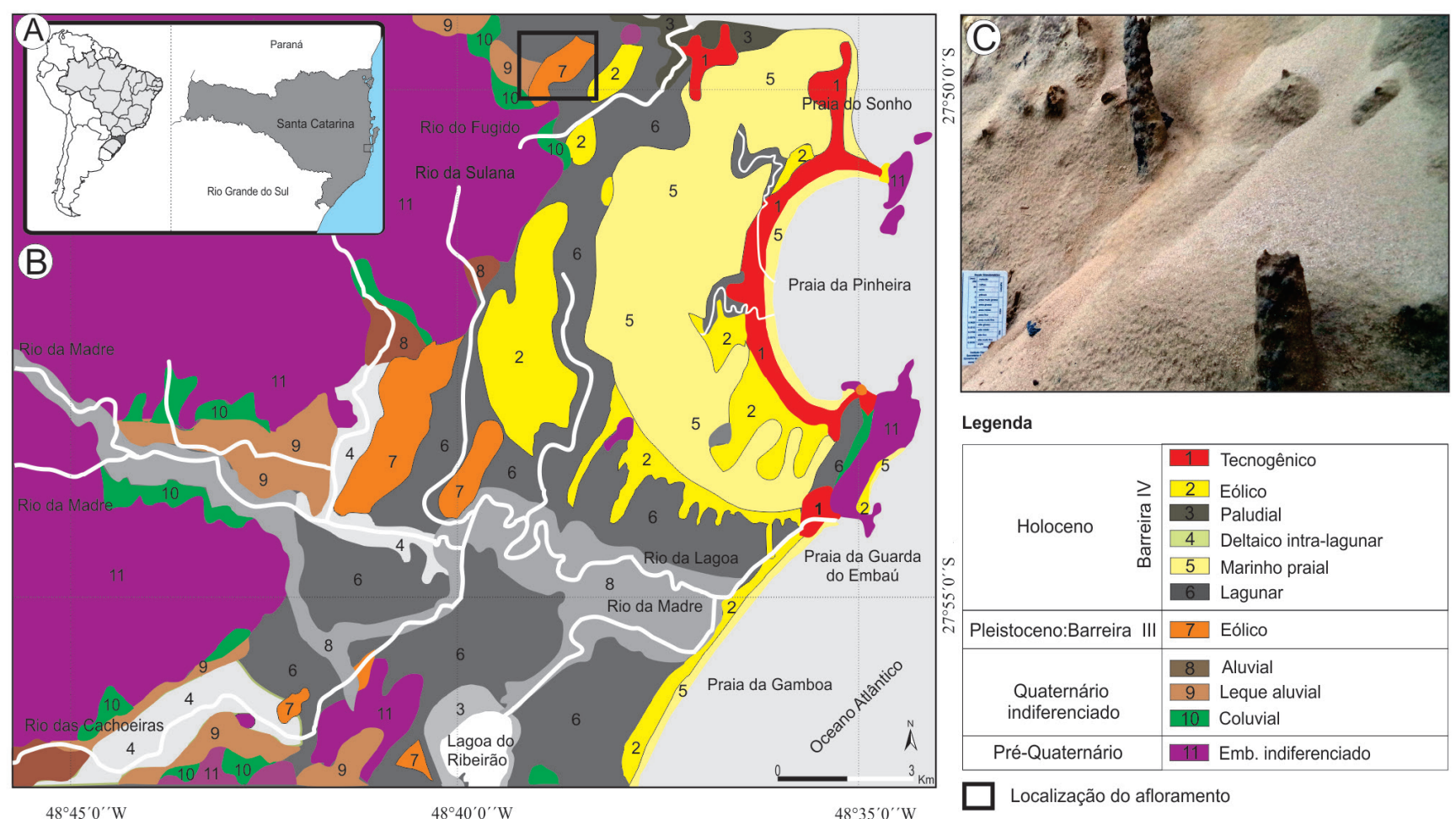

Figura 1. Localização do afloramento estudado. A, mapa de localização da área de estudo; B, mapa geológico com os principais ambientes deposicionais quaternários (modificado de Horn Filho et al., 2012) e C, imagem do afloramento mostrando as galerias que caracterizam Ophiomorpha nodosa em posição vertical.

Figure 1. Location of the studied outcrop. A, location map of the study area; B, geological map with depositional environments during Quaternary (modified from Horn Filho et al., 2012) and C, outcrop image showing Ophiomorpha nodosa in vertical position. 
como interpretação do ambiente deposicional a partir da sua preservação e das estruturas sedimentares associadas, além de suas implicações para a evolução costeira e reconstrução do paleonível marinho.

\section{ÁREA DE ESTUDO}

O afloramento estudado situa-se nas coordenadas $27^{\circ} 49,8^{\prime} \mathrm{S} / 48^{\circ} 38,3^{\prime} \mathrm{W}$, próximo ao Rio Maciambu, com acesso principal pela BR-101, em área pertence à mineração de areia Maciambu, Município de Palhoça, Santa Catarina (Figuras 1A-B). Segundo Horn Filho (2003), a área estudada está inserida no setor central (Setor IV) da Província ou Zona Costeira Catarinense. Os depósitos sedimentares desta região correspondem àqueles associados aos sistemas deposicionais do tipo Laguna-Barreira propostos por Villwock et al. (1986) e Tomazelli \& Villwock (2000) para o Rio Grande do Sul, também utilizados em alguns estudos no litoral sul catarinense por pertencerem à Bacia de Pelotas. Na região, encontram-se depósitos pleistocênicos e holocênicos correspondentes aos ambientes marinho raso, praial, eólico, lagunar e paludial, cujas principais formas de relevo são planícies lagunares, dunas, cordões litorâneos regressivos, terraços e canais fluviais. Os icnofósseis aqui descritos são encontrados em depósitos a noroeste da Praia da Pinheira (Figura 1B). As idades obtidas nos sedimentos indicam que estes depósitos são cronocorrelatos à formação da barreira pleistocênica III, resultado da transgressão marinha ocorrida durante o interglacial, estágio isotópico de oxigênio (MIS) 5, há aproximadamente 125 ka AP (Villwock et al., 1986; Giannini et al., 2007; Hesp et al., 2009; Horn Filho et al., 2012; Dillenburg et al., 2014; Mendes et al., 2015).

Evolutivamente, a Planície Costeira da Pinheira tem sido amplamente discutida como um sistema complexo por apresentar a sobreposição de diversos ambientes deposicionais, que evoluíram durante o Quaternário, principalmente, no que se refere à barreira holocênica e seus ambientes associados (Barboza et al., 2009; Hesp et al., 2009; Amin Jr. \& Dillenburg, 2010; Hein et al., 2013; Dillenburg et al., 2014).
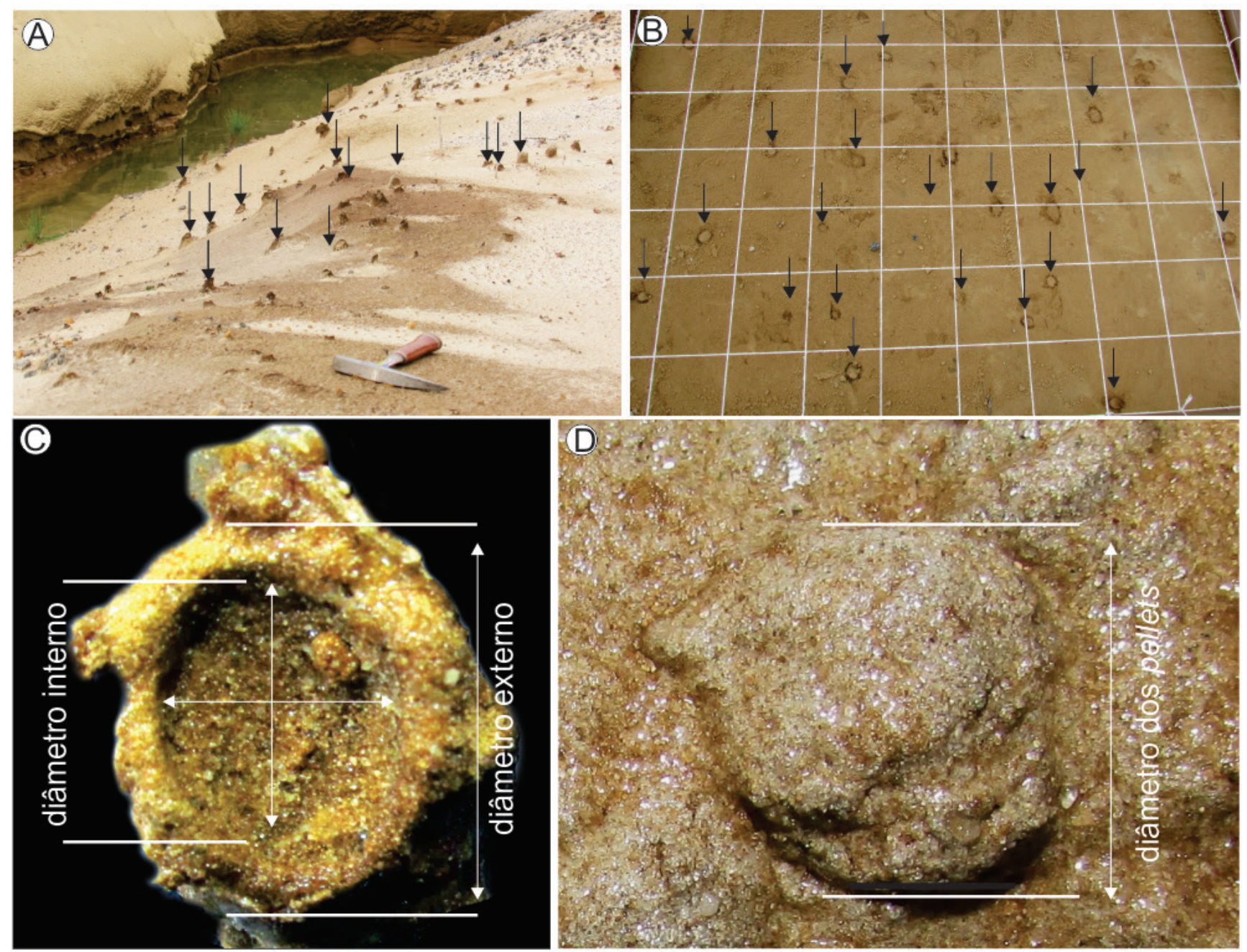

Figura 2. A, galerias em plano horizontal; B, método de contagem por $\mathrm{m}^{2} ; \mathbf{C}$, medidas dos diâmetros interno e externo das galerias; $\mathbf{D}$, medida do diâmetro dos pellets.

Figure 2. A, galleries in plain view; $\mathbf{B}$, counting method per $\mathrm{m}^{2} ; \mathbf{C}$, measure of the internal and external diameter of the galleries; $\mathbf{D}$, measure of pellet diameter. 


\section{MATERIAL E MÉTODOS}

No afloramento, os icnofósseis são visíveis em posição vertical (Figura 1C). Um total de 15 espécimes foi coletado, com a ajuda de pincéis, removendo, cuidadosamente, os sedimentos para posterior análise, e depositados na Coleção de fósseis do Laboratório de Paleontologia da Universidade Federal de Santa Catarina (UFSC-P), sob os números UFSC-P 369 a UFSC-P 383.

Para obtenção da distribuição espacial e densidade, foi contabilizado o número de galerias visíveis no plano horizontal em uma área de $10 \mathrm{~m}^{2}$, com auxílio de quadrículas de 10 x 10 $\mathrm{cm}$ (Figuras 2A-B). A contagem das mesmas foi realizada por meio da adaptação do método utilizado por Botter-Carvalho et al. (2002). Em cada metro quadrado, contabilizou-se o número de galerias visualmente identificáveis, considerando que cada galeria foi tratada como um indivíduo único, como exemplificado na Figura 2B.

Posteriormente, em laboratório, a identificação taxonômica dos icnofósseis foi realizada através da comparação com material bibliográfico (e.g. Kennedy \& MacDougall, 1969; Frey et al., 1978; Griffis \& Suchanek, 1991; Nickell \& Atkinson, 1995; Bromley \& Edkale, 1998; Gibert et al., 2012). Para estabelecer a forma das estruturas, foram tomadas medidas do diâmetro dos pellets e dos diâmetros externo e interno das galerias, com paquímetro de $0,02 \mathrm{~mm}$ de precisão (Figuras 2C-D).

Dados altimétricos em relação ao nível relativo marinho atual (n.r.m.) foram adquiridos por um sistema GNSS, com equipamento Trimble R6 (Receptor multi frequências, RTK, GPS, GLONASS e WAAS/EGNOS), ondulação geoidal de referência: MAPGEO 2010, e corrigidos pelo software Trimble Business Center (TBC).

Abreviatura institucional. UFSC-P, Coleção de Fósseis do Laboratório de Paleontologia da Universidade Federal de Santa Catarina, Florianópolis, SC.

\section{RESULTADOS}

Os icnofósseis analisados consistem apenas das galerias verticais cilíndricas, isoladas e distribuídas aleatoriamente no afloramento (Figura 1C). A densidade de galerias varia de 22 a $51 / \mathrm{m}^{2}$ (média $\left.=32,9 / \mathrm{m}^{2}, \mathrm{n}=10\right)$. Ramificações horizontais, que ocorrem geralmente entre 2 e $4 \mathrm{~m}$ abaixo da superfície do sedimento (Pryor, 1975), não foram observadas. As galerias em seção transversal exibem formato circular a elíptico (Figura 2C). Os diâmetros externos máximos e mínimos medidos foram de 32,82 e 22,86 $\mathrm{mm}$ respectivamente (média $=27,84 \mathrm{~mm}, \mathrm{n}=15$ ). O diâmetro interno das galerias variou de 19,24 a 11,88 mm (média $=15,56 \mathrm{~mm}, \mathrm{n}=15$ ).
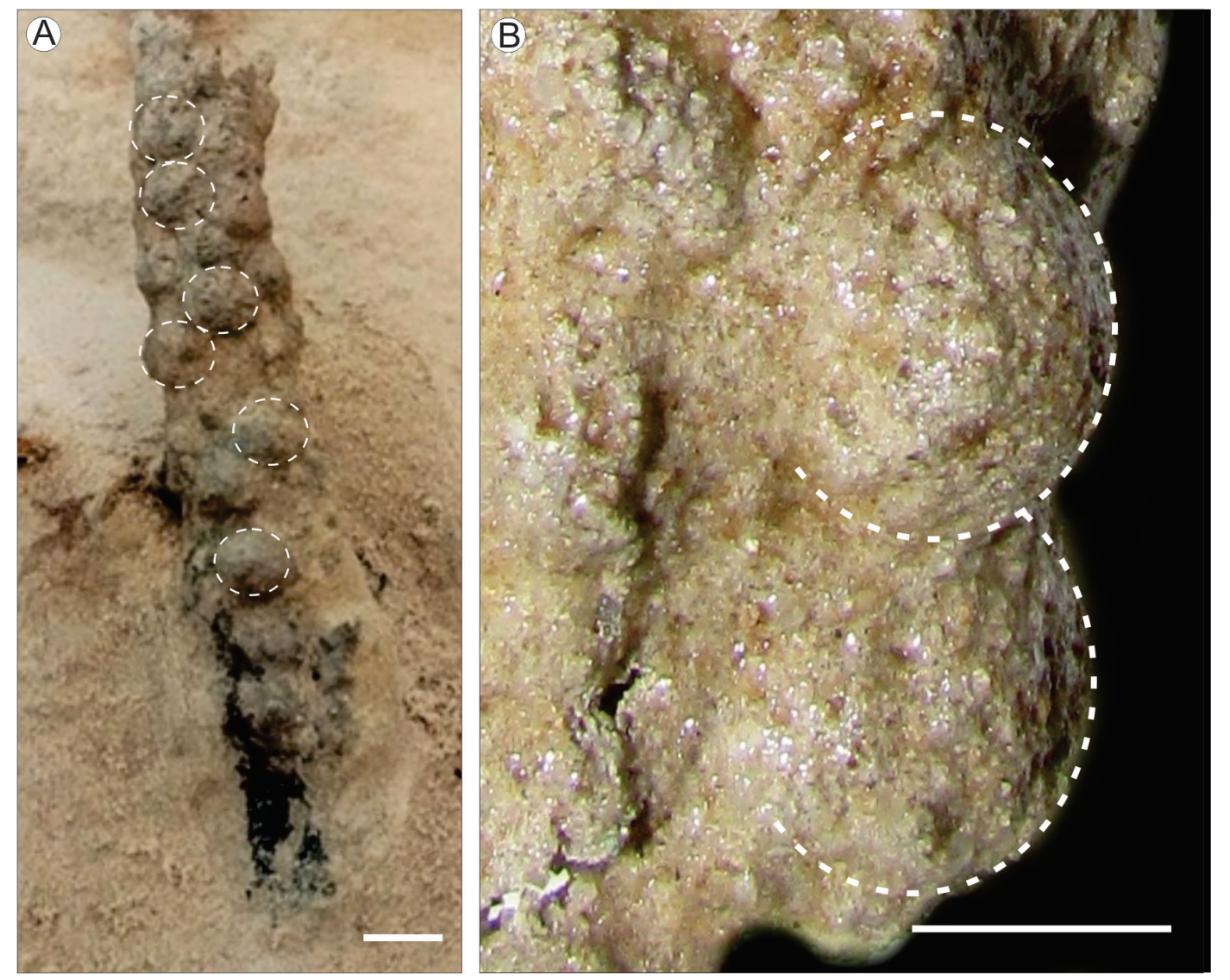

Figura 3. Detalhes da morfologia das galerias fósseis. A, em plano vertical, detalhe da forma e posicionamento dos pellets; B, detalhe dos pellets. Escalas $=10 \mathrm{~mm}$.

Figure 3. Details of the fossil galleries morphology. A, detail in the vertical plane of the shape and position of the pellets; B, detail of the pellets. Scale bars $=10 \mathrm{~mm}$. 


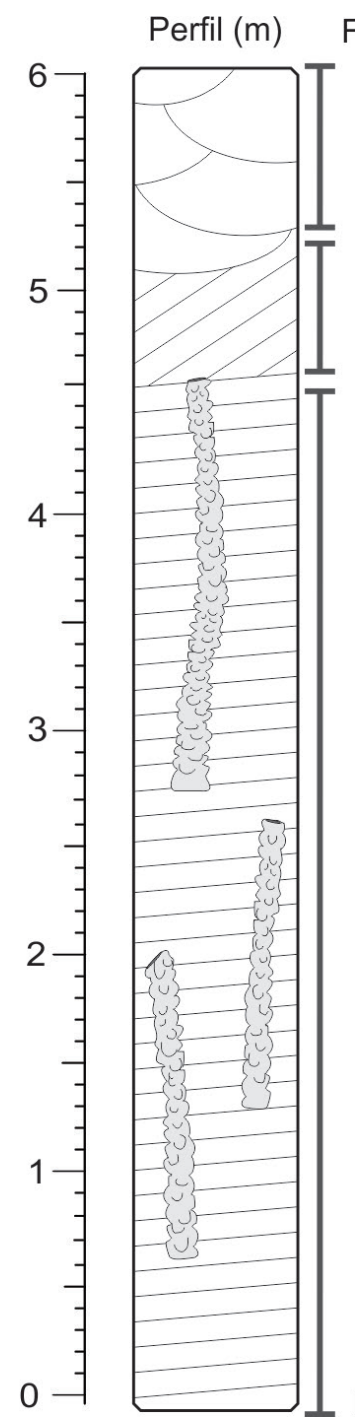

Fácies

(iii)

(ii)
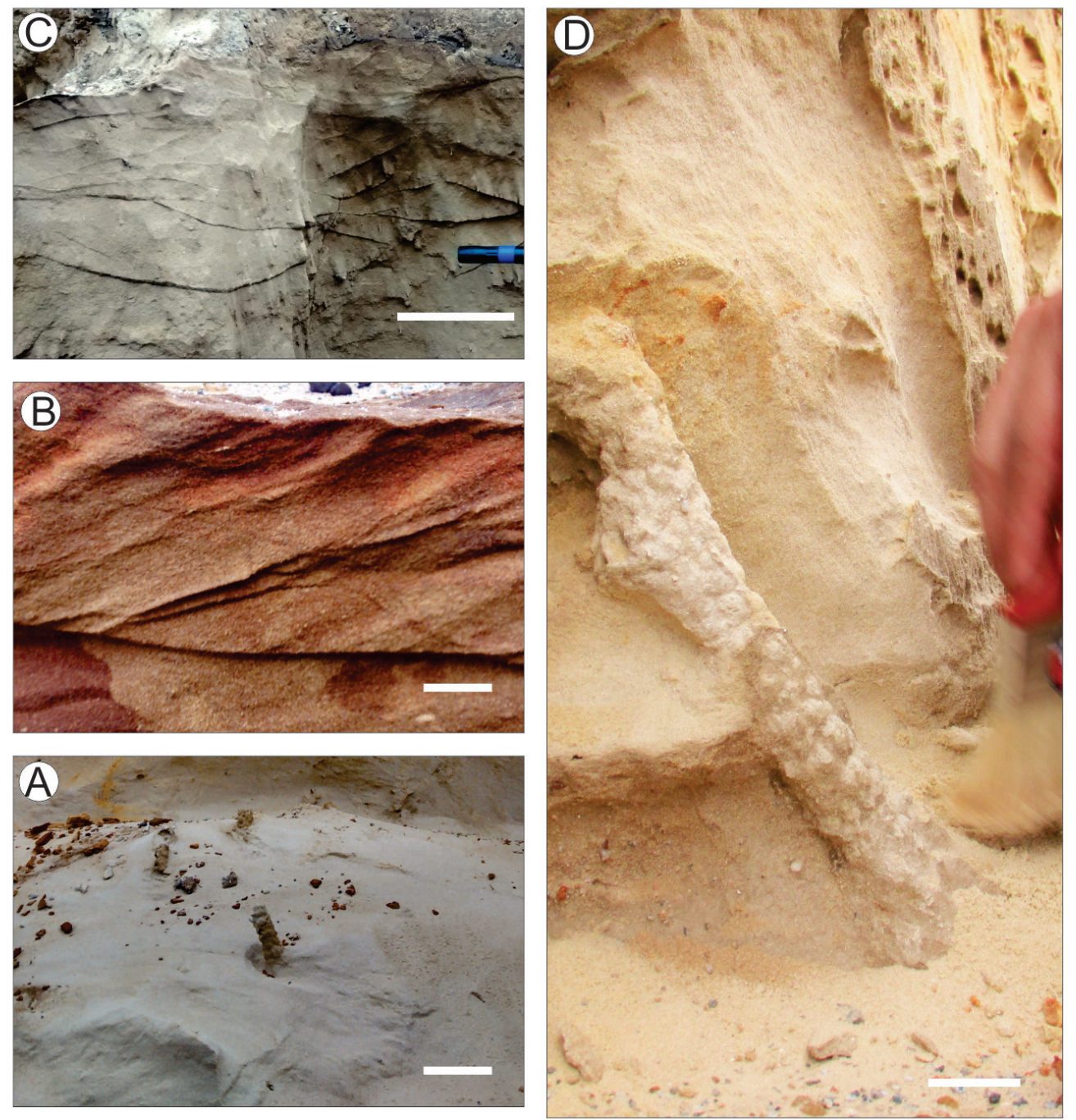

\section{Legenda}

\section{Estratificação plano-paralela $\otimes$ Estratificação cruzada}

Figura 4. Perfil esquemático com as principais estruturas sedimentares primárias do afloramento estudado. A, Areia fina com estrutura plano-paralela (ambiente marinho raso, zona inframareal), fácies (i); B, Areia fina com laminação cruzada (ambiente praial, zona supramareal), fácies (ii); C, sedimentos compostos por areia fina a grossa, com estratificação cruzada-acanalada, fácies (iii); D, Galerias verticais dos icnofósseis. Escalas: A-C = 10 cm; D = 1 cm.

Figure 4. Schematic profile with the main primary sedimentary structures of the studied outcrop. A, fine sediments of beach facies with plane-parallel structure, facies (i); B, fine sediments of aeolian facies with cross lamination, facies (ii); C, fine to coarse sand with trough cross-bedding, facies (iii); D, fossil galleries. Scale bars: $\mathrm{A}-\mathrm{C}=10 \mathrm{~cm} ; \mathrm{D}=1 \mathrm{~cm}$.

A parede externa das galerias é formada por pellets subesféricos constituídos de argila e areia quartzosa de tamanho areia fina a média, distribuídos regularmente na superfície, sem alinhamento ou qualquer tipo de arranjo (Figuras 3A-B). Apresentam diâmetro médio de $8,65 \mathrm{~mm}$, sendo o maior diâmetro encontrado $11,28 \mathrm{~mm}$ e o menor 6,36 $\mathrm{mm}(\mathrm{n}=15)$. A parede interna das galerias é lisa e estas se encontram preenchidas por areia fina (Figura 2C).

No afloramento estudado os depósitos apresentam três fácies principais: (i) fácies de depósitos arenosos com laminação plano-paralela e cruzada de baixo ângulo, medindo aproximadamente $4 \mathrm{~m}$ de espessura e interpretada como ambiente costeiro raso, na qual são encontrados os exemplares de Ophiomorpha nodosa (Figuras 4A, D); (ii) fácies de depósitos arenosos formando sets com estratificação cruzada medindo 0,6 $\mathrm{m}$ de espessura que sugerem migração de barras subaquosas na zona intermareal (Figura 4B); e (iii) fácies de depósitos arenosos com estratificação cruzada acanalada, medindo aproximadamente $1 \mathrm{~m}$ de espessura, indicativa de ambiente de dunas costeira (Figura 4C). O contato entre o topo da fácies (i) e a fácies (ii) é erosivo. Os dados altimétricos mostram que os icnofósseis estão distribuídos verticalmente entre 0 e 4,5 $\mathrm{m}$ acima do n.r.m. 


\section{DISCUSSÃO}

Os caracteres diagnósticos que permitiram atribuir as galerias à icnoespécie Ophiomorpha nodosa incluem o revestimento externo formado por pellets esféricos a ovoides e parede interna lisa (Ophiomorpha do Tipo 1, segundo Kennedy \& MacDougall, 1969). Embora aqueles autores mencionem outros caracteres para esta icnoespécie, como a ramificação das galerias em subsuperfície, em ângulo reto em relação às verticais, formando galerias horizontais com paredes infladas nos pontos de ramificação, estes não puderam ser avaliados devido à ausência de exposição de galerias horizontais no afloramento. Outras duas icnoespécies de Ophiomorpha que ocorrem no Brasil são $O$. irregulaire e $O$. puerilis. No entanto, $O$. irregulaire apresenta pellets cônicos com um arranjo irregular e $O$. puerilis possui pellets alongados e menores do que as outras espécies citadas (Gibert et al., 2006; Leaman et al., 2015; Netto et al., 2017).

Apesar da comparação entre populações sob diferentes condicionantes ambientais ser limitada, estudos a respeito da densidade populacional atual do gênero Callichirus demonstraram médias de 2,9 galerias $/ \mathrm{m}^{2}$ para o Estado de São Paulo (Rodrigues \& Shimizu, 1997) e de 6,07 galerias $/ \mathrm{m}^{2}$ para Pernambuco (Botter-Carvalho et al., 2002). Neste último trabalho, foi registrado um número máximo de 20 galerias $/ \mathrm{m}^{2}$. Por outro lado, dados de densidade de Ophiomorpha nodosa revelaram valores maiores, com média de 16,5 galerias/ $\mathrm{m}^{2}$, em Delaware, nos Estados Unidos (Miller et al., 1998) e de 22 galerias $/ \mathrm{m}^{2}$ em depósitos pleistocênicos da costa atlântica brasileira (Angulo \& Souza, 2014). Os resultados aqui apresentados refletem uma maior densidade média, com 32,9 galerias $/ \mathrm{m}^{2} \mathrm{em} 10 \mathrm{~m}^{2}$. Este número, no entanto, ao invés de representar a densidade real dos icnofósseis, pode estar superestimado devido à sobreposição de galerias ao longo do tempo de deposição da fácies (i), na qual estão preservadas. Neste caso, seriam galerias construídas em intervalos de tempo e níveis diferentes, à medida que a espessura da camada sedimentar aumentava, em resposta à oscilação positiva do nível do mar (criação de espaço de acomodação positivo), indicando time-averaging pela mistura de distintas gerações, como sugerido por Angulo \& Souza (2014).

Por outro lado, a elevada densidade de galerias registrada pode estar relacionada ao contexto ambiental em que foram produzidas. No Golfo do México e costa do estado da Geórgia (EUA), Pryor (1975) registrou densidades de duas a oito galerias $/ \mathrm{m}^{2} \mathrm{em}$ zonas rasas (praia e foreshore) dominadas por ondas, enquanto que, em profundidades de $10 \mathrm{~m}$, a densidade média foi de 20 galerias $/ \mathrm{m}^{2}$. Este valor é comparável aos resultados aqui obtidos, o que indicaria um paleonível marinho (superfície da lâmina d'água) em torno de $10 \mathrm{~m}$ acima do n.r.m, dentro, portanto, dos valores da amplitude da transgressão marinha de $120 \mathrm{ka} \mathrm{AP}$, observados no litoral de Santa Catarina (Giannini et al., 2007; Mendes et al., 2015).

Em depósitos costeiros atuais, Ophiomorpha está distribuído principalmente em praias abertas, na zona situada do meso- ao infralitoral, entre o n.r.m. e a linha da maré baixa, muito raramente ocorrendo acima da linha da maré alta (Weimer \& Hoyt, 1964; Pryor, 1975; Frey et al., 1978). Desta forma, pode-se assumir que a fácies (i) registrada no afloramento representa ambiente correspondente à zona de inframaré. A presença de galerias ao longo de toda a fácies (i) e a ausência de variações faciológicas indicativas de mudança na profundidade e relacionadas à migração horizontal dos ambientes deposicionais (como aumento na quantidade de lama marcando a transição da shoreface superior-foreshore para a shoreface inferior) indica a relativa estabilidade das condições marinhas, em termos de profundidade, à medida que a fácies (i) estava sendo depositada. Este padrão estaria relacionado a uma fase agradacional na evolução dos depósitos costeiros, que marcaria a transição da fase transgressiva para a fase regressiva (Roy et al., 1997). A ausência de Ophiomorpha na fácies (ii) sugere a mudança de contexto deposicional meso-/infralitoral para supralitoral (zona supramareal) e a transição deste para a fácies (iii) (dunas) sobrejacente, indicando o final da transgressão marinha. Padrão similar foi observado no Rio Grande do Sul, onde a fase agradacional seguida de regressão marinha da Barreira II foi inferida a partir dos icnofósseis Ophiomorpha e Rosselia, preservados nos depósitos de upper shoreface-foreshore desta unidade (Lopes et al., 2016).

\section{CONCLUSÕES}

Os icnofósseis foram determinados como Ophiomorpha nodosa através do estudo da morfologia das galerias (pellets, diâmetro externo e interno). A contagem das galerias por $\mathrm{m}^{2} \mathrm{e}$ seu arranjo espacial sugerem relativo time-averanging ou um ambiente de inframaré mais profundo e evidenciam uma fase agradacional da barreira. Estes sedimentos são interpretados como depósitos relacionados à penúltima transgressão marinha, ocorrida há 125 ka AP e correlacionada ao estágio isotópico de oxigênio (MIS) 5. O afloramento estudado indica a transição de um ambiente marinho litorâneo (zona de inframaré), ao qual estão associados os icnofósseis, para um ambiente praial (supramaré), e por fim ambiente costeiro subaéreo (dunas).

\section{AGRADECIMENTOS}

Os autores agradecem ao CNPq (processo 454804/2014-8) pelo financiamento dos trabalhos de campo e a M.L. Martins, proprietário da mineradora, por permitir acesso à área do presente estudo.

\section{REFERÊNCIAS}

Amin Jr., A.H. \& Dillenburg, S.R. 2010. Variation in the granulometric properties of the coastal barrier of Pinheira (SC) during its progradation in the Late Holocene. Quaternary and Environmental Geosciences, 2:25-39. doi:10.5380/abequa. v2i1-2.15130

Angulo, R.J. \& Souza, M.C. 2014. Revisão conceitual de indicadores costeiros de paleoníveis marinhos quaternários no Brasil. Quaternary and Environmental Geosciences, 5:1-32. doi:10.5380/abequa.v5i2.36533 
Barboza, E.G.; Dillenburg, S.R.; Rosa, M.L.C.C.; Tomazelli, L.J. \& Hesp P.A. 2009. Ground-penetrating radar profiles of two Holocene regressive barriers in southern Brazil. Journal of Coastal Research, 56:579-583.

Barboza, J.A.; Viana, M.S.S. \& Neumann, V.H. 2006. Paleoambientes e icnofácies da sequência carbonática da Bacia do Paraíba (Cretáceo-Paleógeno), Nordeste do Brasil. Revista Brasileira de Geociências, 36:73-90. doi:10.25249/03757536.2006363449464

Barreto, A.M.F.; Suguio, K.; Almeida, J.A.C. \& Bezerra, F.M.R. 2002. A presença da icnoespécie Ophiomorpha nodosa Lundgren em rochas sedimentares pleistocênicas da costa norte-riograndense e suas implicações paleoambientais. Revista Brasileira de Paleontologia, 3:17-23.

Botter-Carvalho, M.V.L; Santos, E.J.P. \& Carvalho, P.V.V.C. 2002. Spatial distribution of Callichirus major (Say 1818) (Decapoda: Callianassidae) on a sandy beach, Piedade, Pernambuco, Brazil. Nauplius, 10:97-109.

Bromley, R.E. \& Ekdale, A.A. 1998. Ophiomorpha irregulaire (trace fossil): redescription from the Cretaceous of the Book Cliffs and the Wasatch Plateau, Utah. Journal of Paleontology, 72:773-778. doi:10.1017/S0022336000040476

Bromley, R.G. \& Frey, R.W. 1974. Redescription of the trace fossil Gyrolithes and taxonomic evaluation of Thalassinoides, Ophiomorpha and Spongeliomorpha. Geological Society of Denmark Bulletin, 23:311-335.

Caruso Jr., F.; Suguio, K. \& Nakamura, T. 1999. The Quaternary geological history of the Santa Catarina Southeastern Region (Brazil). Anais da Academia Brasileira de Ciências, 72:257-270. doi:10.1590/S0001-37652000000200011

Dike, E.F. 1972. Ophiomorpha nodosa Lundgren: environmental implications in the Lower Greensand of the Isle of Wight. Proceedings of the Geologists' Association, 83:165-170. doi:10.1016/S0016-7878(72)80003-8

Dillenburg, S.R.; Barboza, E.G.; Hesp, P.A.; Rosa, M.L.C.C.; Angulo, R.J.; Souza, M.C.; Giannini, P.C.F. \& Sawakuchi, A.O. 2014. Discussion: evidence for a transgressive barrier within a regressive strandplain system: implications for complex response to environmental change (Hein et al., 2013). Sedimentology, 61:2205-2212. doi:10.1111/sed.12132

Dominguez, J.M.L. \& Bittencourt, A.C.S. 2009. Geologia. In: V. Hatje \& J.B. Andrade (eds.) Baía de todos os Santos, EDUFBA, p. 27-66.

Fernandes, A.C.C.; Borghi, L.; Carvalho, I.S. \& Abreu, C.J. 2002. Guia dos Icnofósseis de Invertebrados do Brasil. Rio de Janeiro, Interciência, $260 \mathrm{p}$.

Frey, R.W.; Howard, J.D. \& Pryor, W.A. 1978. Ophiomorpha: its morphologic taxonomic and environmental significance. Palaeogeography, Palaeoclimatology, Paleoecology, 23:199229. doi:10.1016/0031-0182(78)90094-9

Gandini, R. \& Netto, R.G. 2012. Ophiomorpha from Lower Permian sandstones of Southern Brazil. In: INTERNATIONAL CONGRESS ON ICHNOLOGY, 3, 2012. Abstract Book, Newfoundland, p. 35.

Giannini, P.C.F.; Sawakuchi, A.O.; Martinho, C.T.; Tatumi, S.H. 2007. Eolian depositional episodes controlled by Late Quaternary relative sea level changes on the Imbituba-Laguna coast (southern Brazil). Marine Geology, 237:143-168. doi:10.1016/j.margeo.2006.10.027

Gibert, J.M.; Curran, H.A.; Netto, R.G.; Tognoli, F.M.W. \& Belaustegui, Z. 2012. Burrowing capabilities of juvenile thalassinideans: paleobiological significance of Ophiomorpha puerilis from the Pleistocene of the Bahamas and Southern
Brazil. In: INTERNATIONAL CONGRESS ON ICHNOLOGY, 2, 2012. Abstract Book, Newfoundland, p. 25.

Gibert, J.M.; Netto, R.G.; Tognoli, F.M.W. \& Grangeiro, M.E. 2006. Commensal worm traces and possible juvenile thalassinidean burrows associated with Ophiomorpha nodosa, Pleistocene, southern Brazil. Palaeogeography, Palaeoclimatology, Palaeoecology, 230:70-84. doi:10.1016/j.palaeo.2005.07.008

Gingras, M.K.; Räsänen, M. \& Ranzi, A. 2002. The significance of bioturbated inclined heterolithic stratification in the Southern part of the Miocene Solimões Formation, Rio Acre, Amazonia, Brazil. Palaios, 17:591-601. doi:10.1669/08831351(2002)017<0591:TSOBIH>2.0.CO;2

Griffis, R.B. \& Suchanek, T.H. 1991. A model of burrow architecture and trophic modes in thalassinidean shrimp (Decapoda: Thalassinidea). Marine Ecology Progress Series, 79:171-183. doi:10.3354/meps079171

Häntzschel, W. 1975. Trace fossils and problematica. In: C. Teichert (ed.) Treatise on Invertebrate Paleontology, Geological Society of America and University of Kansas, p. 1-269.

Hein, C.J.; Fitz Gerald, D.M.; Cleary, W.J.; Albernaz, M.B.; Menezes, J.T. \& Klein, A.H. 2013. Evidence of transgressive barrier and regressive strandplain system: implication of complex coastal response to environmental change. Sedimentology, 60:469-502. doi:10.1111/j.1365-3091.2012.01348.x

Hesp, P.A.; Giannini, P.C.F.; Martinho, C.T.; Silva, G.M. \& Asp, N.E. 2009. The Holocene barrier systems of the Santa Catarina Coast, Southern Brazil. In: P.A. Hesp \& S.R. Dillenburg (eds.) Geology and Geomorphology of Holocene Coastal Barrier of Brazil, Berlin, Springer, p. 93-133 (Lecture Notes in Earth Sciences 107). doi:10.1007/978-3-540-44771-9_4

Horn Filho, N.O. 2003. Setorização da Província Costeira de Santa Catarina em base aos aspectos geológicos, geomorfológicos e geográficos. Geosul, 18:71-98.

Horn Filho, N.O. et al. 2012. Mapa geológico da planície costeira das folhas Paulo Lopes (SG-22-Z-D-V-4) e Imbituba (SH-22$X-B-I I-2)$, Santa Catarina, Brasil. Florianópolis, Universidade Federal de Santa Catarina.

Horn Filho, N.O. \& Simó, D.H. 2008. The Upper Pleistocene of São Francisco do Sul Island Coastal Plain: geomorphologic, sedimentologic and evolutive aspects. Brazilian Journal of Oceanography, 56:179-187. doi:10.1590/S167987592008000300003

Howard, J.D. 1972. Trace fossils as criteria for recognizing shorelines in stratigraphic record. In: J.K. Rigby \& W.K. Hamblin (eds.) Recognition of ancient sedimentary environments, Tulsa, Society for Sedimentary Geology, p. 215-225 (Special Publication 16). doi:10.2110/pec.72.02.0215

Kennedy, W.J. \& MacDougall, J.D.S. 1969. Crustacean burrows in the Weald Clay (Lower Cretaceous) of south-eastern England and their environmental significance. Palaeontology, 12:459-471.

Leaman, M.; McIlroy, D.; Herringshawa, L.G.; Boyd, C. \& Callow, R.H.T. 2015. What does Ophiomorpha irregulaire really looks like? Palaeogeography, Palaeoclimatology, Palaeoecology, 439:38-49. doi:10.1016/j.palaeo.2015.01.022.

Lopes, R.P.; Caron, F. \& Dillenburg, S.R. 2016. Stratigraphic significance of Rosselia and Ophiomorpha trace fossils in a Pleistocene coastal barrier of Rio Grande do Sul, Brazil. In: CONGRESSO BRASILEIRO DE GEOLOGIA, 48, 2016. Resumos, Porto Alegre, SBG.

Lopes, R.P.; Dillenburg, S.R.; Schultz, C.L.; Ferigolo, J.; Ribeiro, A.M.; Pereira, J.C.; Holanda, E.C.; Pitana, V.G. \& Kerber, L. 2014. The sea-level highstand correlated to marine isotope stage (MIS) 7 in the coastal plain of the state of Rio Grande do Sul, 
Brazil. Anais da Academia Brasileira de Ciências, 86:1573-595. doi:10.1590/0001-3765201420130274

Mendes, V.R.; Giannini, P.C.F.; Guedes, C.C.F.; DeWitt, R. \& Andrade, H.A.A. 2015. Central Santa Catarina coastal dunefields chronology and their relation to relative sea level and climatic changes. Brazilian Journal of Geology, 45:79-95. doi:10.1590/2317-4889201530143

Miller, M.F.; Curran, H.A. \& Ronald, L. 1998. Ophiomorpha nodosa in estuarine sands of the lower Miocene Calvert Formation at the Pollack Farm Site, Delaware. Newark, Delaware Geological Survey, p. 41-46 (Special Publication 21).

Netto, R.G.; Curran, H.A.; Belaústegui, Z. \& Tognoli, F.M.W. 2017. Solving a cold case: new occurrences reinforce juvenile callianassids as the Ophiomorpha puerilis tracemakers. Palaeogeography, Palaeoclimatology, Palaeoecology, 475:93105. doi:10.1016/j.palaeo.2017.03.013

Netto, R.G.; Gibert, J.M.; Belaustegui, Z.; Curran, H.A. \& Tognoli, F.M.W. 2012. Ophiomorpha nodosa from Southern Atlantic Pleistocene deposits with comparison to modern callianassid burrows of the southernmost coast of Brazil. In: INTERNATIONAL CONGRESS ON ICHNOLOGY, 2, 2012. Abstract Book, Newfoundland, p. 64.

Netto, R.G. \& Rossetti, D.F. 2003. Ichnology and salinity fluctuations: a case study from the early Miocene (Lower Barreiras Formation) of São Luís Basin, Maranhão, Brazil. Revista Brasileira de Paleontologia, 6:5-18.

Nickell, L.A. \& Atkinson, R.J.A. 1995. Functional morphology of burrows and trophic modes of three thalassinidean shrimp species, and a new approach to the classification of thalassinidean burrow morphology. Marine Ecology Progress Series, 128:181197. doi:10.3354/meps 128181

Pryor, W.A. 1975. Biogenic sedimentation and alteration of argillaceous sediments in shallow marine environments. Geological Society of America Bulletin, 86:1244-1254. doi:10.1130/0016-7606(1975)86<1244:BSAAOA>2.0.CO;2

Rodrigues, S.A. \& Shimizu, R.M. 1997. Autoecologia de Callichirus major (Say,1818). Oecologia Brasiliensis, 3:155-170. doi:10.4257/oeco.1997.0301.10

Rosa, M.L.C.C.; Barboza, E.G.; Abreu, V.S.; Tomazelli, L.J. \& Dillenburg, S.R. 2017. High frequency sequences in the Quaternary of Pelotas Basin (coastal plain): a record of degradational stacking as a function of longer-term base-level fall. Brazilian Journal of Geology, 47:183-207. doi:10.1590/2317-4889201720160138

Rossetti, D.F. \& Góes, A.M. 2009. Marine influence in the Barreiras Formation, State of Alagoas, northeastern Brazil. Anais da Academia Brasileira de Ciências, 81:741-755. doi:10.1590/ S0001-37652009000400012
Roy, P.S.; Cowell, P.J.; Ferland, M.A. \& Thom, B.G. 1997. Wavedominated coasts. In: R.W.G. Carter \& C.D. Wodroffe (eds) Coastal Evolution - Late Quaternary Shoreline Morphodynamics, Cambridge University Press, p. 121-186.

Stewart, D.J. 1978. Ophiomorpha: a marine indicator? Proceedings of the Geologists' Association, 89:33-41. doi:10.1016/S00167878(78)80019-4

Suguio, K.; Bezerra, F.H.R. \& Barreto, A.M.F. 2011. Luminescence dated Late Pleistocene wave-built terraces in northeastern Brazil. Anais da Academia Brasileira de Ciências, 83:907-920. doi:10.1590/S0001-37652011005000010

Suguio, K. \& Martin, L. 1976. Presença de tubos fosseis de "Callianassa" nas formações quaternárias do litoral paulista e sua utilização na reconstrução paleoambiental. Boletim do Instituto de Geociências, 7:17-26. doi:10.11606/issn.23168978.v7i0p17-25

Tomazelli, L.J. \& Dillenburg, S.R. 2007. Sedimentary facies and stratigraphy of a last interglacial coastal barrier in south Brazil. Marine Geology, 244:33-45. doi:10.1016/j.margeo.2007.06.002

Tomazelli, L.J \& Villwock, J.A. 2000. O Cenozóico no Rio Grande do Sul: geologia da planície costeira. In: M. Holz \& L.F. De Ros (eds.) Geologia do Rio Grande do Sul, IGeo/UFRGS, p. 375-406.

Tomazelli, L.J.; Villwock, J.A.; Loss, E.L. \& Denhardt, E.A. 1982. Caracterização de um depósito praial pleistocênico na Província Costeira do Rio Grande do Sul. In: CONGRESSO BRASILEIRO DE GEOLOGIA, 32, 1982. Anais, Salvador, SBG, p. 1514-1523.

Vieira, M.M.; de Ros, L.F. \& Bezerra, F.H.R. 2007. Lithofaciology and palaeoenvironmental analysis of Holocene beachrocks in Northeastern Brazil. Journal of Coastal Research, 23:15351548. doi:10.2112/05-0562.1

Vieira, C.V.; Possamai, T. \& Horn Filho, N.O. 2011. Geologia costeira da Ilha de São Francisco do Sul/SC. In: CONGRESSO DA ASSOCIAÇÃO BRASILEIRA DE ESTUDOS DO QUATERNÁRIO, 13, 2011. Anais, Armação dos Búzios, ABEQUA, p. 45-58.

Villwock, J.A.; Tomazelli, L.J.; Loss, E.L.; Dehnhardt, E.A.; Horn, N.O.; Bachi, F.A. \& Dehnhardt, B.A. 1986. Geology of the Rio Grande do Sul coastal province. Quaternary of South America and Antarctic Peninsula, 4:79-97.

Weimer, J.K.T. \& Hoyt, J.H. 1964. Burrows of Callianassa major Say, geologic indicators of littoral and shallow neritic environments. Journal of Paleontology, 38:761-767.

Received in 04 August, 2017; Accepted in 30 March, 2018. 